\title{
Polarization Mode Locking in Erbium Doped Fiber Ring Lasers
}

\author{
M. Romagnoli, A. Tajani, M. Tamburrini, S. Wabnitz, B. Daino \\ Fondazione Ugo Bordoni, Via B. Castiglione 59,00142 Roma, Italy \\ F. Fontana, N. Manfredini, G. Grasso \\ Societd Cavi Pirellli, Viale Sarca 2, 20126 Milano, Italy \\ P. Franco, M. Midrio \\ Dipartimento di Elettronica ed Informatica, Università di Padova, Via Gradenigo 6/A, 35131, \\ Padova, Italy
}

Introduction: Mode-locking (ML) with erbium doped fiber lasers permits to combine the wide spectral bandwidth of the gain medium with the anomalous group velocity dispersion of the fiber. As a result, soliton pulse shaping plays a key role in the ML dynamics [1-2]. By combining ultrashort soliton amplification and soliton switching [3], it has been demonstrated that ML erbium doped fiber lasers may operate in the femtosecond regime [4]. These ML sources are intrinsically self-starting and unstable [5], and the origin of the observed complex temporal behavior has been recently ascribed to soliton resonances with the laser cavity length [6].

In this work we present results of experimental studies of the role of polarization rotation as a switching mechanism [7] in both active and passive ML in erbium doped fiber ring lasers. In the active case, by controlling the state of polarization we observed the generation of ML 100 femtosecond pulse trains, with repetition rates as high as $300 \mathrm{GHz}$. In the passive case, ML is self-starting and we observed the generation of multiple pulses with different wavelengths and polarization states [8]. Here the control of the state of polarization permits the selection of the output wavelengths.

Experiment: The experimental configurations for active and passive $\mathrm{ML}$ in erbium doped fiber ring lasers are shown in fig.(1). In both cases, gain is provided by a $10 \mathrm{~m}$ long erbium doped fiber (aluminium codoped), with the numerical aperture $\mathrm{NA}=0.2$, and the absorption of $5.5 \mathrm{~dB} / \mathrm{m}$ at the wavelength $\lambda=1532 \mathrm{~nm}$. Whereas the cutoff wavelength is $900 \mathrm{~nm}$, which permits single mode propagation of the $980 \mathrm{~nm}$ pump from a dichroic coupler. The maximum power from the semiconductor pump laser was equal to $39 \mathrm{~mW}$. Unidirectional operation was ensured by means of a polarization insensitive optical isolator. Active $\mathrm{ML}$ was initiated by $\mathrm{LiNbO}_{3}$ phase modulator, and the state of polarization after the isolator was manipulated by means of an all-fiber polarization controller. In the passive case, a bulk polarizing beam splitter was employed in order to reject one 
polarization component (vertical) from the cavity. Whereas in the active case polarization discrimination was achieved through a directional coupler. In the passive configuration, we also monitored the polarization state inside the cavity (horizontal) by means of a beam sampler. In either case, the average output power from the lasers ranged from 0.2 to $2 \mathrm{~mW}$. The output light was detected with fast photodiodes and sampling oscilloscopes, which in some cases permitted to directly measure the repetition rate of the circulating trains. In the femtosecond ML regime, we have performed autocorrelations of the output pulse trains after an erbium doped fiber amplifying stage (with a linear gain of $20 \mathrm{~dB}$ ).

Results: In the active case, as the modulator was turned on the cw laser oscillation broke up into a regular train of $\mathrm{ML}$ pulses. The repetition rate of the train was equal to the fundamental frequency of the cavity $(7.5 \mathrm{MHz})$, irrespectively to some extent of the modulation frequency. The time duration of the ML train in the envelope was $5 \mathrm{~ns}$. Figure (2) shows the autocorrelation of the ML pulses, which reveals the 100 fs full width of the pulses (possibly time compressed along the amplifier). The inset in figure (1) shows that the repetition rate of the ML train was as high as 300 $\mathrm{GHz}$. The control of the state of polarization in the ring was critical for obtaining ultrashort pulse ML. Nevertheless, once it was achieved the ML was relatively stable.

In the passive case, self-starting ML was observed to prevail over $\mathrm{cw}$ oscillation. The temporal characteristics of the generated pulses consisted of a superposition pulses circulating at the fundamental cavity frequency and at higher harmonics. Therefore we have characterized the laser output in the spectral domain. We found that the laser tends to mode lock pulses with different wavelengths and polarizations. Figure (3) shows a case of single-frequency emission, which is accompanied by several satellite sidebands. From the main spectrum of fig.(3) we infer a ML pulse width of the order of $30 \mathrm{ps}$, which would rule out the soliton resonances with the loop length [6] as a possible mechanism for sideband generation. Moreover, here the sidebands are equally spaced whereas soliton resonance leads to wavelength separations that are proportional to the square root of the sideband order [6].

Figure (4) shows dual-frequency ML in the passive ring laser. Here we have removed the polarizer since only the beam splitter introduces polarization sensitive losses in the cavity. The polarization decomposition that is shown in fig.(4) follows the directions which are either parallel or orthogonal to the entry plane of incidence of the splitter. As it can be seen, the polarization state of the two peaks is different. Moreover, the sidebands only appear in one polarization. The observed anisotropy disappears reducing the pump power.

ML was not only limited to pulses with two different frequencies. By simply rotating the polarization controller, it was possible to introduce several polarized optical paths in the cavity which leads to multiple frequency ML (see fig.(5)) [8]. 
Conclusions: We have studied polarization induced passive and active $\mathrm{ML}$ in a simple erbium doped fiber ring laser configuration. In the active case, we have demonstrated the generation of ultrahigh repetition rate femtosecond pulse trains. In the case of self-starting passive ML, we have revealed the generation of polarized sidebands and multiple frequency ML.

Acknowledgements: This work was carried out in the framework of the agreement between Fondazione Ugo Bordoni and the Italian P.T. Administration.

\section{References}

[1] J. D. Kafka, T. Baer, and D. W. Hall, Opt. Lett. 14, 1269 (1989).

[2] K. Smith, J. R. Armitage, R. Wyatt, and N. J. Doran, Electron. Lett. 26, 1149 (1990).

[3] A. G. Bulushev, E. M. Dianov, and O. G. Okhotnikov, Opt. Lett. 15, 968 (1990).

[4] I. N. Duling, Electron. Lett. 27, 544 (1991).

[5] R. P. Davey, N. Langford, and A. I. Ferguson, Electron. Lett. 27, 1257 (1991); D. J. Richardson, R. I. Laming, D. N. Payne, V. J. Matsas, and M. W. Phillips, Electron. Lett. 27, $1451(1991)$.

[6] N. Pandit, D. U. Noske, S. M. J. Kelly, and J. R. Taylor, Electron. Letters 28, 455 (1992).

[7] M. Hofer, M. E. Fernmann, F. Haberl, M. H. Ober, and A. J. Schmidt, Opt. Lett. 16, 502 (1991).

[8] J. B. Schlager, S. Kawanishi, M. Saruwatari, Electron. Lett. 27, 2073 (1991).

\section{Active configuration}

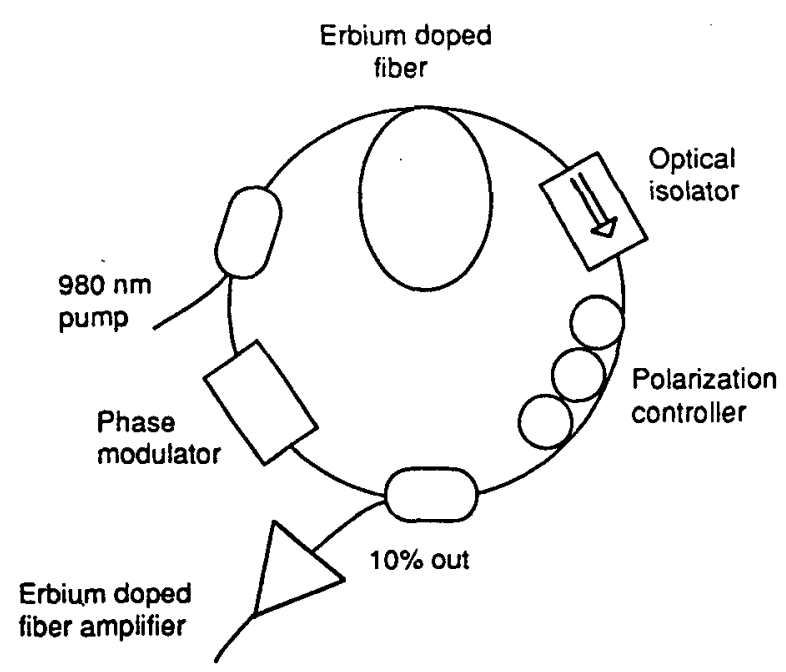

\section{Self-starting configuration}

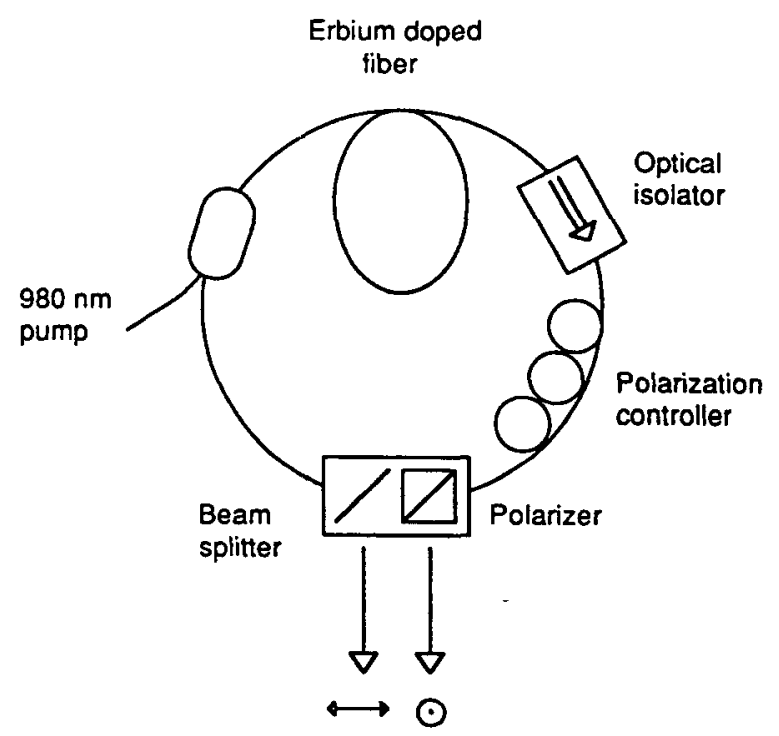

Figure 1: Schematic of active and passive polarization mode-locking with erbium doped fiber ring lasers. 

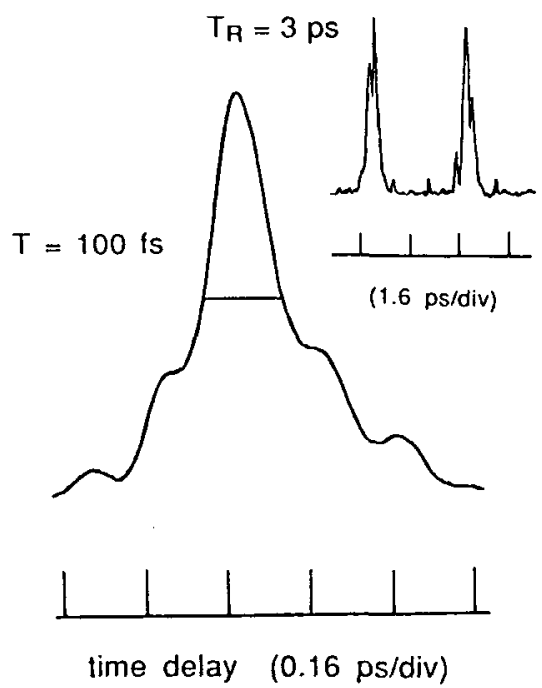

Figure 2: Autocorrelation of $100 \mathrm{fs}$ output pulse from actively mode locked erbium fiber ring laser. The inset shows the autocorrelation of the pulse train with 3 ps repetition rate.

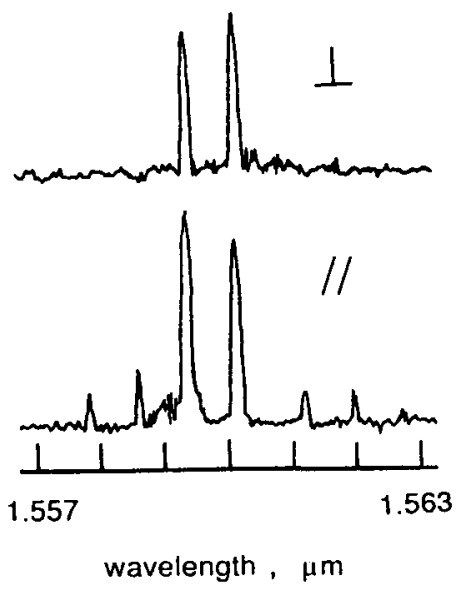

Figure 4: Polarization decomposition of dual frequency passive mode locking at high pump powers.

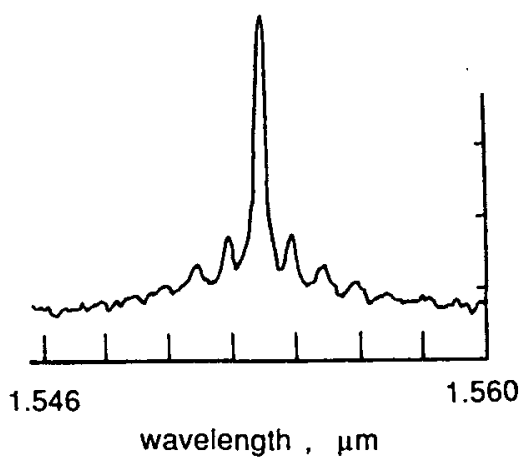

Figure 3: Multiple sideband generation from self-starting passive mode locked laser.
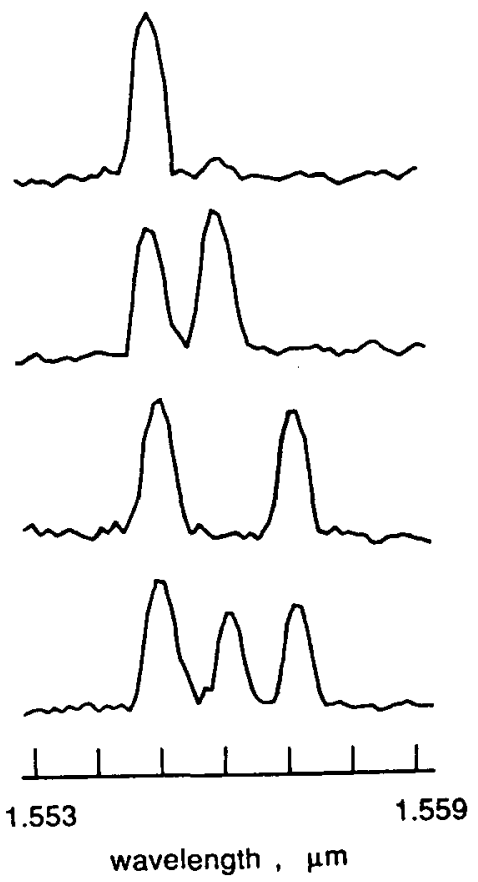

Figure 5: Birefringence-controlled triple frequency passive mode locking. 